\title{
Smart Sensor Systems for Extremely Harsh Environments
}

\author{
H. Kappert' ${ }^{1}$ S. Schopferer², R. Döring ${ }^{3}$, S. Ziesche ${ }^{4}$, A. Olowinsky ${ }^{5}$, F. Naumann ${ }^{6}$, M. Jägle ${ }^{7}$, \\ A. Ostmann ${ }^{8}$ \\ ${ }^{1}$ Fraunhofer Institute for Microelectronic Circuits and Systems IMS \\ 2 Fraunhofer Institute for High-Speed-Dynamics, Ernst-Mach-Institut EMI \\ ${ }^{3}$ Fraunhofer Institute for Electronic Nanosystems ENAS \\ ${ }^{4}$ Fraunhofer Institute for Ceramic Technologies and Systems IKTS \\ ${ }^{5}$ Fraunhofer Institute for Laser Technology ILT \\ 6 Fraunhofer Institute for Microstructure of Materials and Systems IMWS \\ ${ }^{7}$ Fraunhofer Institute for Physical Measurement Techniques IPM \\ ${ }^{8}$ Fraunhofer Institute for Reliability and Microintegration IZM \\ holger.kappert@ims.fraunhofer.de
}

\begin{abstract}
Summary:
Sensors systems are key elements for capturing environmental properties and are increasingly important in industry 4.0 for the intelligent control of processes. However, under harsh operating conditions like high temperatures, high mechanic load or aggressive environments, standard electronics cannot be used. Eight Fraunhofer institutes have therefore bundled their competencies in sensors, microelectronics, assembly, board design, laser applications and reliability analysis to establish a technology platform for sensor systems working under extreme conditions.
\end{abstract}

Keywords: harsh environments, high temperature, ceramic sensors, integrated circuits, assembly

\section{Introduction}

Reliable sensor systems are increasingly important in the industry for the intelligent control of industrial processes. Fig. 1 shows a typical example of a generic sensor system. A number of sensors take information from the environment and transform them into electrical signals. A dedicated signal conditioning circuitry performs e.g. offset compensation, amplification, filtering and analog to digital conversion. Further processing is often done by a microcontroller with calculation and storage capacity for identification and calibration data. Standard interfaces like CAN or RS485 are commonly used for the connection to a higher level system making them capable to interact with Industry 4.0 processes.

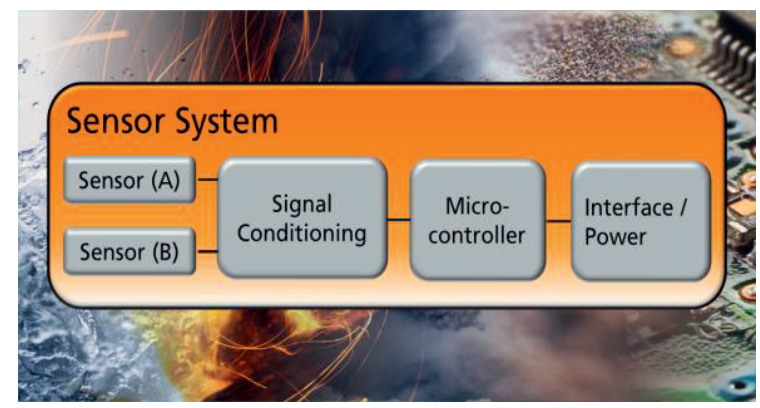

Fig. 1: Typical sensor system with analog and digital signal conditioning and interfacing.
While in many everyday objects highly integrated sensor systems are already state of the art, the situation in an industrial environment is clearly different. Up to now, the use of sensor systems here was often impossible, because the extreme ambient conditions of industrial processes like high operating temperatures, strong mechanical load or a humid or chemically aggressive environment do not allow reliable operation of sensitive electronic components.

However, due to the need for energy and resource savings as well as featuring environmentally friendly processes, detailed process control is necessary and monitoring of vital process parameters under extreme conditions becomes more and more essential. Applications can be found in various fields like steel treatment, jet engines, stationary turbines as well as deep drilling for oil, gas or geothermal energy.

\section{Scope of the Lighthouse Project 'eHarsh'}

The realization of such sensor systems requires a multidisciplinary approach including e.g. the design of reliable sensor elements, high temperature integrated circuits, and appropriate assembly and housing techniques. Therefore eight Fraunhofer Institutes have concentrated 
their competencies and have initiated the Fraunhofer Lighthouse Project 'eHarsh'.

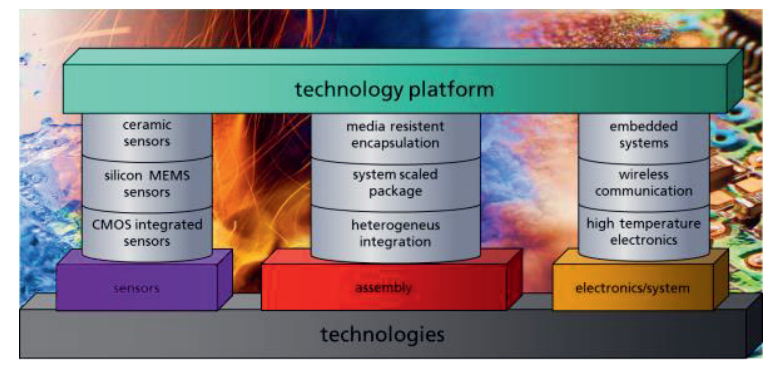

Fig. 2: Technological basis of the 'eHarsh' project.

'eHarsh' is a common approach involving all necessary technologies for the design of robust sensor systems for harsh environments. Two demonstrators are planned to show the successful implementation of the technology platform: the first is a pressure sensor system with sensor, microelectronics and dedicated ceramic board design for temperatures up to $300{ }^{\circ} \mathrm{C}$ with peek temperatures at the tip of the sensor up to $500{ }^{\circ} \mathrm{C}$ for avionic applications. The second is a sensor system for geothermal applications at temperatures up to $300{ }^{\circ} \mathrm{C}$ and under high pressure.

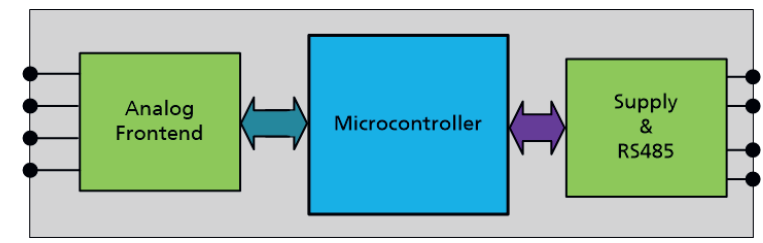

Fig. 3: Integrated chipset for the 'eHarsh' demonstrators.

In the project various technologies and components have been investigated like high temperature ceramic based pressure and temperature sensors, an integrated chipset (Fig. 3) for signal conditioning and processing featuring operating temperatures of up to $300{ }^{\circ} \mathrm{C}$ as well as various assembly and board technologies. Special focus is on the application of well-known technologies even for high temperature operation like flip chip assembly in combination with ceramic circuit boards and the application of embedding in combination with high temperature printed circuit boards. Special emphasis was on hermetic sealing of the sensor using laser beam micro welding of the metallic casing and glassbased connection between ceramic and metal.

All developments have been gone along with comprehensive characterization as well as simulation and analysis for reliability. In addition, new test and characterization methods have been investigated.
Fig. 4 shows a model of the high temperature sensor for avionic applications with integrated electronics.

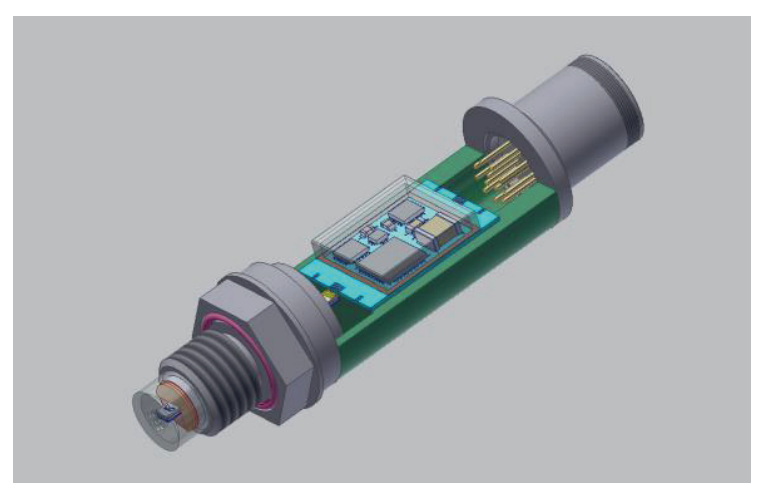

Fig. 4. Demonstrator for avionic applications.

\section{Current status}

In the first part of the project, the development of the envisaged technologies has been performed and verified. In the current second phase the demonstrators are assembled and first measurements have been made. In the remaining project time, demonstrators will be finished and extensively tested under the harsh conditions of the envisaged applications.

\section{Acknowledgements}

This work was supported as a Fraunhofer LIGHTHOUSE PROJECT.

\section{References}

[1] H. Kappert: 'Sensor Systems for Extreme harsh Environments', Plenary Session, International Conference and Exhibition on High Temperature Electronics (HiTEC 2018), Albuquerque, New Mexico, USA

[2] S. Ziesche, et al.: 'On-turbine multisensors based Hybrid Ceramic Manufacturing Technology', High Temperature Electronics Network (HiTEN 2019), Oxford, UK

[3] H. Kappert, et al.: 'High Temperature SOI CMOS Technology and Circuit realization for Applications up to $300^{\circ} \mathrm{C}$ ', IEEE International Symposium on Circuits and Systems (ISCAS), pp. 11621165, May 2015 